\title{
Differentiation of Isomeric C8-Substituted Alkylaniline Adducts of Guanine by Electrospray Ionization and Tandem Quadrupole Ion Trap Mass Spectrometry
}

\author{
Linge Li and M. Paul Chiarelli \\ Department of Chemistry, Loyola University, Chicago, Illinois, USA
}

Paula S. Branco and Alexandra M. Antunes

Departamento de Química, Centro de Química Fina e Biotecnologia, Faculdade de Ciências e Tecnologia, Universidade Nova de Lisboa, Caparica, Portugal

\section{Matilde Marques and Luísa L. Gonçalves}

Centro de Química Estrutural, Complexo I, Instituto Superior Técnico, Lisboa, Portugal

Frederick A. Beland

Division of Biochemical Toxicology, National Center for Toxicological Research, Jefferson, Arkansas, USA

Product ion spectra from thirteen C8-substituted alkylaniline adducts of guanine and deoxyguanosine were generated using electrospray ionization and quadrupole ion trap mass spectrometry and studied to investigate the possibility of differentiating isomeric adduct structures based upon the relative abundances of fragment ions derived from the alkylanilinemodified guanine bases $\left(\mathrm{BH}_{2}^{+}\right.$ions). The structural discrimination of the $\mathrm{BH}_{2}^{+}$ions formed by attachment of isomeric alkylanilines to the $\mathrm{C} 8$ position of guanine is a challenging problem because the ions tend to yield product ion spectra that are qualitatively identical upon collisional activation. In this study, a statistical method, referred to as a similarity index, was used to compare the product ion spectra of isomeric $\mathrm{BH}_{2}^{+}$ions and differentiate their structures. All the adducts investigated could be distinguished from SIs calculated using 5-6 product ions. These results suggest that a searchable database of product ion spectra may be created and used to characterize DNA adducts from aromatic amines whenever they are detected at levels amenable to mass spectral analysis. (J Am Soc Mass Spectrom 2003, 14, 1488-1492) (c) 2003 American Society for Mass Spectrometry

I nterest in the analysis of carcinogen-modified nucleic acids is motivated by the belief that covalent adducts play an important role in the initiation of cancer. Aromatic amines are one class of carcinogens to which human exposure is widespread. The structural characterization of DNA adducts formed by single-ring aromatics is of interest because they constitute the largest arylamine fraction in tobacco smoke [1] and several are rodent carcinogens [2,3].

Product ion studies of carcinogen-modified nucleobases synthesized in vitro have been conducted to establish structure-specific fragmentation pathways

Published online October 22, 2003

Address reprint requests to Dr. M. P. Chiarelli, Department of Chemistry, Loyola University, 1068 W. Sheridan Rd., Chicago, IL 60626, USA. E-mail: mchiare@luc.edu that may serve as signatures for the structures of adducts isolated from in vivo samples. Product ion studies have been successful at differentiating arylamine-deoxyguanosine isomers whose structures differ by the sites of attachment of the arylamine and guanine fragments. Isomers formed by arylamine attachment to the C8, $N^{2}[4], N 1$, and $O^{6}$ [5] positions of guanine all show structure-specific fragmentation pathways. However, adduct structures formed by the attachment of isomeric alkylanilines to the $\mathrm{C} 8$ atom of guanine are difficult to differentiate because they produce product ion mass spectra that are qualitatively identical.

In this study we demonstrate the structural differentiation of thirteen C8-substituted deoxyguanosine and guanine adducts from methyl-, dimethyl-, and ethylanilines by using ESI and quadrupole ion trap mass 


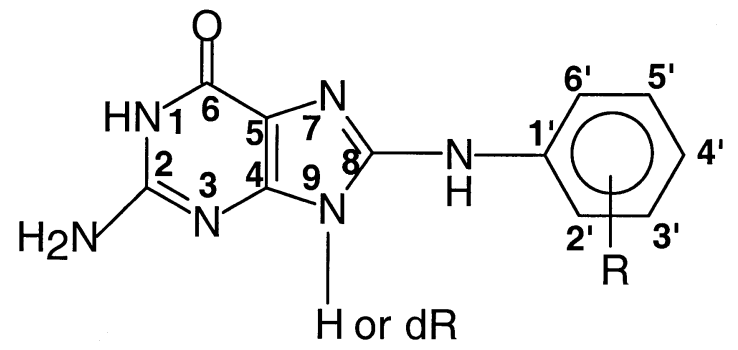

Figure 1. Structure and numbering system of the adducts used in this study. $\mathrm{R}=2$-Me (1), 3-Me (2), 4-Me (3), 2,3-diMe (4), 2,4-diMe (5), 2,5-diMe (6), 2,6-diMe (7,8), 3,4-diMe (9), 3,5-diMe (10), 2-EtA (11), 3-EtA (12), 4-EtA (13). Adducts 1, 2, 3, 5, and 8 were deoxyguanosine derivatives. All other adducts were guanine derivatives.

spectrometry (QITMS) and calculation of a similarity index (SI) [6]. Our results suggest that ESI and QITMS may be used to develop a database of product ion spectra to facilitate the identification of unknown DNA adducts.

\section{Experimental}

\section{Synthesis of C8-Substituted Guanine-Alkylaniline Adducts}

The C8-substituted guanine adducts were synthesized as described $[7,8]$, with minor modifications. The reaction mixture was evaporated and re-dissolved in a minimal amount of $2 \mathrm{M} \mathrm{HCl}$. The C8-substituted guanine adducts were enriched by solid phase extraction and isolated by HPLC using a water:methanol gradient [7]. The concentrations of the guanine adducts were estimated from their UV absorbance using the $\lambda_{\max }$ and $\varepsilon$ values of the corresponding C8-substituted deoxyguanosine adducts $[9,10]$. The yields for the eight different dimethyl and ethyl aniline adducts synthesized in this study varied from $3-7 \%$.

The synthesis and characterization of the five C8substituted deoxyguanosine adducts have been described [10-12]. The structures and numbering system of all thirteen adducts investigated are summarized in Figure 1.

\section{Mass Spectrometry}

Molecular weight and product ion mass spectra were acquired with a ThermoFinnigan Advantage (San Jose, CA) LCQ ion trap mass spectrometer. The same instrument parameter values were used for all adducts under study. The source-induced dissociation voltage was set to $20 \%$ of its maximum value to optimize the abundances of the $\mathrm{BH}_{2}^{+}$ions formed from the deoxyguanosine adducts. The tandem mass spectra energy parameter was set to $35 \%$ of its maximum value for studies of the methylaniline adducts and 38\% for studies of the dimethyl- and ethylaniline adducts. Product ion spectra of all the $\mathrm{BH}_{2}^{+}$ions were acquired by direct infusion of low picomolar amounts of sample. A 1:1:1 mixture of water:methanol:acetonitrile was used as the mobile phase. For comparative purposes, eight product ion spectra were acquired for each compound.

\section{Results and Discussion}

Tandem mass spectra of the $\mathrm{BH}_{2}^{+}$ions formed from thirteen different C8-substituted alkylaniline adducts of guanine and deoxyguanosine were studied with the intent of differentiating the structures of isomers using ESI and QITMS. The product ion spectra of the $\mathrm{BH}_{2}^{+}$ ions did not show any fragmentation patterns which, upon visual inspection, allowed isomeric adducts to be distinguished. Therefore, we attempted to differentiate the structures of isomeric $\mathrm{BH}_{2}^{+}$ions through the calculation of a SI, using the ion abundance values observed in their product ion spectra.

\section{Fragmentation of Alkylaniline Adducts}

The deoxyguanosine adducts used in this study produced $(\mathrm{M}+\mathrm{H})^{+}$ions almost exclusively under full-scan conditions. The product ion spectra of the protonated molecules showed the $\mathrm{BH}_{2}^{+}$ion formed by glycosidic cleavage as the only significant fragment. Therefore, the source-induced dissociation parameter was adjusted to maximize the production of the $\mathrm{BH}_{2}^{+}$ions observed in the full scans for product ion analysis. Representative product ion spectra of these $\mathrm{BH}_{2}^{+}$ions are shown in Figure 2. In all instances, the product ion spectra of the C8-substituted guanines exhibited the same five fragmentation pathways (bond cleavages labeled a-e in Figure 2 that have been described previously [13]. Briefly, all the $\mathrm{BH}_{2}^{+}$ions analyzed in this study underwent neutral losses of $17\left(\mathrm{NH}_{3}\right), 42\left(\mathrm{CH}_{2} \mathrm{~N}_{2}\right)$, and 70 $\left(\mathrm{C}_{2} \mathrm{H}_{2} \mathrm{~N}_{2} \mathrm{O}\right)$ mass units (bond cleavages labeled a-c, respectively) in order of decreasing relative abundance, through bond cleavages in the six-membered ring of guanine. In addition, all adducts showed ions at $m / z 140$ and 165, formed through the loss of the alkylaniline ring system (bond cleavages $d$ and e, respectively). The three ethylaniline adducts exhibited an additional fragment at $\mathrm{m} / \mathrm{z} 152$, corresponding to protonated guanine, that was not observed in the product ion spectra of the mono- and dimethylaniline adducts.

\section{Comparison of Product Ion Spectra from Methylaniline Adducts}

Initially, two statistical methods were tested for their ability to differentiate the structures of the three methylaniline adducts from deoxyguanosine (Compounds 1-3) through comparison of the product ion spectra of the corresponding $\mathrm{BH}_{2}^{+}$ions. As indicated above, the SI 


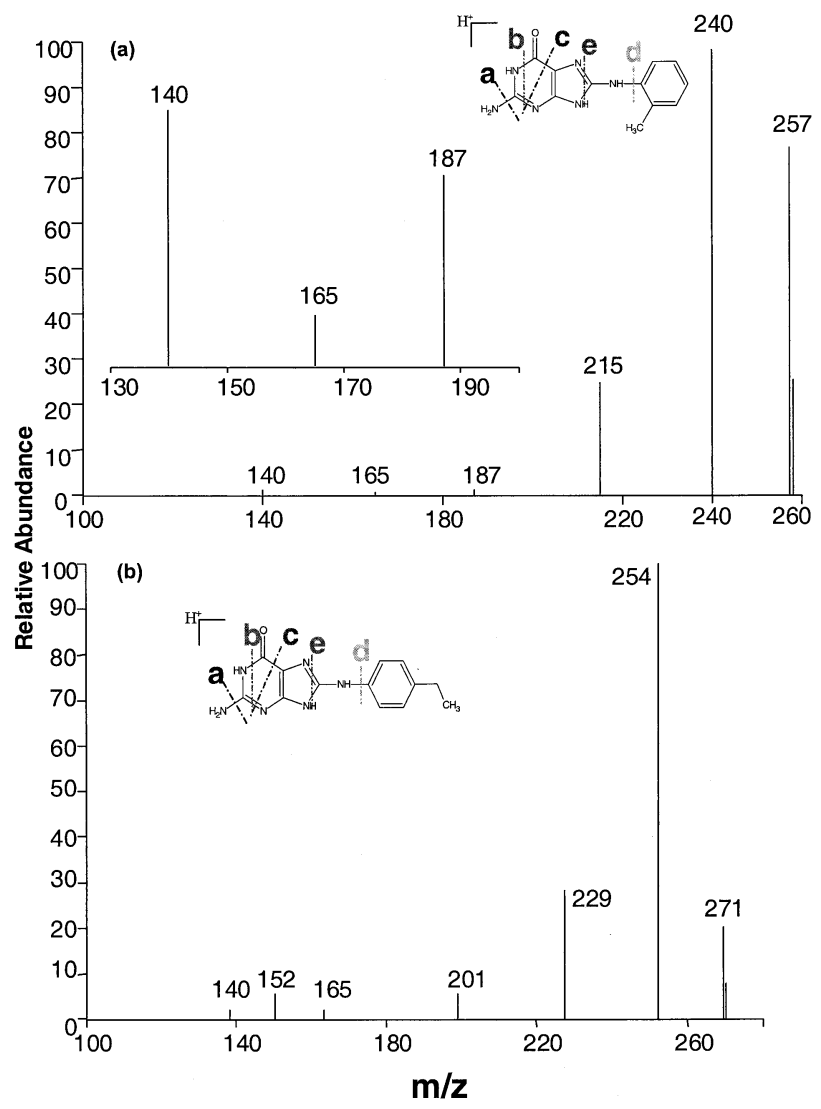

Figure 2. Representative ESI /QIT product ion mass spectra of the $\mathrm{BH}_{2}^{+}$ions formed from (a): dG-C8-2-MeA (1) The $m / z$ 130-200 region is expanded (inset) to show the ions at $m / z 140, m / z 165$, and m/z 187; (b): G-C8-2-EtA (13).

was developed as a quantitative means of testing the similarity of two product ion spectra [6].

$$
S I=\sqrt{\frac{\left[\left(i-i_{0}\right) / i_{0} \times 100\right]^{2}}{N}}
$$

The SI is calculated according to eq 1 , where $i$ and $i_{0}$ are the relative abundances of two ions of the same $\mathrm{m} / \mathrm{z}$ value and $\mathrm{N}$ is the number of product ion signals that are being compared. Modification of the quotient in eq 1 has been suggested [14, 15], with the purpose of eliminating the arbitrary designation of $i$ and $i_{0}$ (eq 2).

$$
S I=\sqrt{\frac{\left[\left(i-i_{0}\right) /\left(i+i_{0}\right) \times 100\right]^{2}}{N}}
$$

Spectral contrast angle $(\theta)$ is another method used to compare product ion spectra [16]. In mass spectrometry, this parameter, calculated from eq 3 , has been used to search computer databases of unknown ESI spectra [17] and tandem mass spectra of peptides [18].

$$
\cos \theta=\frac{\sum_{i} a_{i} b_{i}}{\sum_{i} a_{i}^{2} \sum_{i} b_{i}^{2}}
$$

Here, $a_{i}$ and $b_{i}$ represent the relative abundances of product ions of the same $\mathrm{m} / \mathrm{z}$ value in the two product ion spectra being compared. The terms in the numerator and denominator are summed over the number of $\mathrm{m} / \mathrm{z}$ values being compared. The spectral contrast angle and modified SI methods were tested for their ability to distinguish the structures of the $\mathrm{BH}_{2}^{+}$ions formed from the three $\mathrm{C} 8$-substituted methylaniline adducts. The relative abundances of all five product ions mentioned above were used for these calculations. The average relative abundances observed of these product ions are summarized in Table 1. The self- and inter-adduct SIs are given in Table 2 . The self-SI or contrast angle (e.g., Compound $\mathbf{1}$ versus $\mathbf{1}$ ) is the average value calculated using product ion spectra acquired from the same compound. The inter-adduct SI or contrast angles (e.g., Compound 1 versus 2) were calculated by comparing the product ion mass spectra acquired from different compounds. The smaller the SI or contrast angle, the greater the similarity of the product ion spectra being compared; thus, isomeric structures are considered differentiated when the self-SIs calculated for each compound are substantially smaller than the SIs obtained when spectra acquired from the different compounds are compared $(>\sigma)$. For example, the self-SIs associated with the 2-MeA and 4-MeA adducts are $6 \pm 2$ and $5 \pm$ 1 , respectively, whereas the SI obtained when the spectra of these two adducts are compared is $18 \pm 3$ (Table $1)$.

In this study, the relative standard deviations associated with the contrast angle calculations $(38-75 \%)$ were found to be much larger than those associated with the SI calculation $(9-25 \%$; Table 1) as can be deduced form the data presented in Table 1. These results suggest that the modified SI may be more useful than the spectral contrast angle for distinguishing the structures of isomeric $\mathrm{BH}_{2}^{+}$ions derived from C8substituted deoxyguanosine methylaniline adducts.

Table 1. Average relative abundances and standard deviations of the product ions observed in the $\mathrm{CAD}$ spectra of the $\mathrm{BH}_{2}^{+}$ions at $\mathrm{m} / \mathrm{z} 257$ derived from the C8-substituted methylaniline adducts of guanine

\begin{tabular}{lccccc}
\hline Compound & $\mathrm{m} / \mathrm{z} 140$ & $\mathrm{~m} / \mathrm{z} 165$ & $\mathrm{~m} / \mathrm{z} 187$ & $\mathrm{~m} / \mathrm{z} 215$ & $\mathrm{~m} / \mathrm{z} 240$ \\
\hline \hline dG-C8-2-MeA (1) & $6 \pm 1$ & $1.3 \pm 0.3$ & $3.7 \pm 0.5$ & $30 \pm 1$ & 100 \\
dG-C8-3-MeA (2) & $4.9 \pm 0.3$ & $0.2 \pm 0.1$ & $3.2 \pm 0.3$ & $27.6 \pm 0.8$ & 100 \\
dG-C8-4-MeA (3) & $5.0 \pm 0.4$ & $1.0 \pm 0.3$ & $3.6 \pm 0.4$ & $28 \pm 1$ & 100 \\
\hline
\end{tabular}


Table 2. Average Modified Similarity Indices (SI) and spectral contrast angles ( $\theta)$ and standard deviations calculated from the product ion spectra of methylaniline adducts (1-3)

\begin{tabular}{lcccrr}
\hline Adduct spectra compared & Self-SI & Self- $\theta$ & $\begin{array}{c}\text { Adduct spectra } \\
\text { compared }\end{array}$ & SI & $34 \pm 3$ \\
\hline \hline DG-C8-2-MeA (1) & $6 \pm 2$ & $0.8 \pm 0.3$ & $(\mathbf{1}, \mathbf{2})$ & $1.8 \pm 0.8$ \\
DG-C8-3-MeA (2) & $4 \pm 1$ & $0.7 \pm 0.4$ & $(\mathbf{1}, \mathbf{3})$ & $18 \pm 3$ & $1.2 \pm 0.5$ \\
DG-C8-4-MeA (3) & $5 \pm 1$ & $0.8 \pm 0.6$ & $(\mathbf{2}, \mathbf{3})$ & $28 \pm 4$ & $1.1 \pm 0.5$ \\
\hline
\end{tabular}

\section{Comparison of Dimethyl- and Ethylaniline Adduct Spectra Using the Modified SI}

The results obtained with the methylaniline adducts prompted us to test the modified SI as a means of differentiating structurally similar adducts from a larger set of alkylanilines. Product ion spectra were acquired of the $\mathrm{BH}_{2}^{+}$ions derived from 10 different dimethyl- and ethylaniline adducts of guanine and deoxyguanosine (Figure 1). The relative abundances of six product ions were used in these calculations and their average relative abundances are summarized in Table 3. Eight product ion spectra were acquired for each adduct, and a self-SI was calculated for each adduct using these spectra. SIs were then calculated through pairwise comparison of spectra from different adducts (Table 4). The results shown in Table 2 suggest that each of the tested isomers could be distinguished from the others in a database search.

As a control, we compared the product ion spectra of the $\mathrm{BH}_{2}^{+}$ions derived from $N$-(guan-8-yl)-2,6-dimethylaniline (7) and $\mathrm{N}$-(deoxyguanosin-8-yl)-2,6-dimethylaniline (8). These experiments were conducted to investigate how sensitive fluctuations in product ion abundance might be to different ion generation mechanisms and/or adduct isolation procedures. The comparison is of interest because C8-substituted guanine and deoxyguanosine adducts from the same aromatic amine should yield identical $\mathrm{BH}_{2}^{+}$ions for product ion analysis. Thus, the SI obtained from the cross-comparison of spectra acquired from the two adducts ( 7 and 8$)$ should be similar to the self-SIs obtained in the comparison of product ion spectra generated from each individual $\mathrm{BH}_{2}^{+}$ion. However, the ion generation mechanism may affect the $\mathrm{SI}$, since the $\mathrm{BH}_{2}^{+}$ion from
Compound 7 was produced by direct protonation, whereas the $\mathrm{BH}_{2}^{+}$ion from Compound 8 was produced by glycosidic cleavage of the deoxyguanosine moiety. The self-SIs obtained for the $\mathrm{BH}_{2}^{+}$ions from Compounds 7 and 8 are $2 \pm 1$ and $4 \pm 2$, respectively (Table 2). The SI obtained when product ion spectra from each of these compounds were contrasted is $5 \pm 2$ (Table 2), suggesting that there were no significant differences in the ion abundances observed in the product ion spectra of these two $\mathrm{BH}_{2}^{+}$ions.

\section{Conclusion}

The product ion spectra of the $\mathrm{BH}_{2}^{+}$ions generated from thirteen C8-substituted alkylaniline adducts of guanine and deoxyguanosine have been studied with the aim of differentiating isomeric structures. The modified SI was found to be a better statistical method than the spectral contrast angle for this purpose. The structures of all isomeric $\mathrm{BH}_{2}^{+}$ions investigated in the present study could be differentiated based upon the calculation of the similarity index using five to six product ions. These results suggest that a searchable database of product ion spectra may be created and used to identify unknown guanine adducts from aromatic amines when they are detected at levels that allow structural information through mass spectral analysis.

\section{Acknowledgments}

This work was supported in part by a grant from the American Cancer Society (RPG-00-233-01-CNE). The authors thank the National Cancer Institute Repository at the Midwest Research Institute (Kansas City, MO) for the guanine-3-oxide. They thank Dr.

Table 3. Average relative abundances and standard deviations of the product ions formed by the CAD of the $\mathrm{BH}_{2}^{+}$ion at $m / z 271$ ion derived from the C8-substituted dimethyl- and ethylanine adducts of guanine

\begin{tabular}{lcccccc}
\hline Compound & $\mathrm{m} / \mathrm{z} 140$ & $\mathrm{~m} / \mathrm{z} 152$ & $\mathrm{~m} / \mathrm{z} 165$ & $\mathrm{~m} / \mathrm{z} 201$ & $\mathrm{~m} / \mathrm{z} 229$ & $\mathrm{~m} / \mathrm{z} 254$ \\
\hline \hline G-C8-2,3-diMeA (4) & $4.1 \pm 0.2$ & $\mathrm{~N} / \mathrm{A}$ & $2.4 \pm 0.2$ & $6.3 \pm 0.3$ & $25.1 \pm 0.6$ & 100 \\
dG-C8-2,4-diMeA (5) & $3.6 \pm 0.3$ & $\mathrm{~N} / \mathrm{A}$ & $9.1 \pm 0.3$ & $5.8 \pm 0.3$ & $26 \pm 1$ \\
G-C8-2,5-diMeA (6) & $4.1 \pm 0.3$ & $\mathrm{~N} / \mathrm{A}$ & $2.5 \pm 0.2$ & $6.1 \pm 0.3$ & $24.7 \pm 0.8$ & 100 \\
G-C8-2,6-diMeA (7) & $5.0 \pm 0.2$ & $\mathrm{~N} / \mathrm{A}$ & $11.3 \pm 0.3$ & $7.1 \pm 0.3$ & $26.9 \pm 0.8$ & 100 \\
dG-C8-2,6-diMeA (8) & $5.0 \pm 0.3$ & $\mathrm{~N} / \mathrm{A}$ & $10.7 \pm 0.6$ & $7.2 \pm 0.5$ & $26.7 \pm 0.9$ & 100 \\
G-C8-3,4-diMeA (9) & $3.4 \pm 0.6$ & $\mathrm{~N} / \mathrm{A}$ & $3.3 \pm 0.8$ & $5.5 \pm 0.7$ & $24 \pm 2$ & 100 \\
G-C8-3,5-diMeA (10) & $4.0 \pm 0.3$ & $\mathrm{~N} / \mathrm{A}$ & $0.5 \pm 0.1$ & $6.1 \pm 0.3$ & $24.6 \pm 0.4$ & 100 \\
G-C8-2-EtA (11) & $2.8 \pm 0.4$ & $2.4 \pm 0.6$ & $1.7 \pm 0.4$ & $4.8 \pm 0.8$ & $26 \pm 2$ & 100 \\
G-C8-3-EtA (12) & $3.4 \pm 0.2$ & $0.3 \pm 0.1$ & $0.5 \pm 0.1$ & $5.6 \pm 0.3$ & $25.2 \pm 0.7$ & 100 \\
G-C8-4-EtA (13) & $2.3 \pm 0.2$ & $5.7 \pm 0.3$ & $2.1 \pm 0.3$ & $5.7 \pm 0.3$ & $27.5 \pm 0.8$ & 100 \\
\hline
\end{tabular}


Table 4. Average Modified Similarity Indices (SI) and standard deviations calculated from the product ion spectra of the dimethyl and ethylaniline adducts (4-13)

\begin{tabular}{|c|c|c|c|c|c|c|c|c|c|c|}
\hline Adducts & 4 & 5 & 6 & 7 & 8 & 9 & 10 & 11 & 12 & 13 \\
\hline G-C8-2,3-diMeA (4) & $4 \pm 1$ & & & & & & & & & \\
\hline dG-C8-2,4-diMeA (5) & $26 \pm 2$ & $4 \pm 1$ & & & & & & & & \\
\hline G-C8-2,5-diMeA (6) & $12 \pm 2$ & $25 \pm 1$ & $4 \pm 2$ & & & & & & & \\
\hline G-C8-2,6-diMeA (7) & $28 \pm 2$ & $20 \pm 2$ & $32 \pm 1$ & $2 \pm 1$ & & & & & & \\
\hline dG-C8-2,6-diMeA (8) & $27 \pm 2$ & $19 \pm 3$ & $32 \pm 2$ & $5 \pm 2$ & $4 \pm 2$ & & & & & \\
\hline G-C8-3,4-diMeA (9) & $13 \pm 4$ & $18 \pm 3$ & $14 \pm 2$ & $31 \pm 3$ & $27 \pm 3$ & $6 \pm 1$ & & & & \\
\hline G-C8-3,5-diMeA (10) & $37 \pm 2$ & $40 \pm 1$ & $32 \pm 1$ & $45 \pm 1$ & $44 \pm 1$ & $35 \pm 2$ & $5 \pm 1$ & & & \\
\hline G-C8-2-EtA (11) & $45 \pm 3$ & $49 \pm 2$ & $43 \pm 1$ & $54 \pm 3$ & $53 \pm 3$ & $45 \pm 3$ & $49 \pm 2$ & $7 \pm 1$ & & \\
\hline G-C8-3-EtA (12) & $53 \pm 2$ & $55 \pm 1$ & $50 \pm 2$ & $58 \pm 1$ & $58 \pm 1$ & $52 \pm 2$ & $42 \pm 1$ & $44 \pm 3$ & $7 \pm 2$ & \\
\hline G-C8-4-EtA (13) & $45 \pm 1$ & $48 \pm 1$ & $43 \pm 1$ & $54 \pm 1$ & $53 \pm 1$ & $44 \pm 2$ & $50 \pm 1$ & $24 \pm 1$ & $46 \pm 2$ & $4 \pm 2$ \\
\hline
\end{tabular}

Ilan Vidavsky at Washington University (St. Louis, MO) for the spreadsheet used for the spectral contrast angle calculations.

\section{References}

1. Patrianakos, C.; Hoffman, D. Chemical Studies on Tobacco Smoke LXIV. On the Analysis of Aromatic Amines in Cigarette Smoke. J. Anal. Toxicol. 1979, 3, 150-154.

2. Koujitani, T.; Yasuhara, K.; Toyosawa, K.; Shimada, A.; Onodera, H.; Takagi, H.; Tamura, T.; Hirose, M.; Mitsumori, K. Immunohistochemical and Ultrastructural Studies of 2,6-Dimethylaniline-Induced Nasal Proliferative Lesions in a Rat TwoStage Nasal Carcinogenesis Model Initiated with N-Bis(2Hydroxypropyl)Nitrosamine. Toxicol. Pathol. 2001, 29, 300307.

3. U. S. National Toxicology Program. Toxicology and Carcinogenesis Studies of 2,6-Xylidine (2,6-Dimethylaniline) (CAS No. 87-62-7) in Charles River CD Rats (Feed Studies), Technical Report Series No. 278, 1990, National Toxicology Program, Research Triangle Park, NC.

4. Chiarelli, M. P.; Lay, J. O., Jr. Differentiation of Isomeric C8and $\mathrm{N}^{2}$-Deoxyguanosine Adducts of 2-Acetylaminofluorene by Fast Atom Bombardment and Tandem Mass Spectrometry. J. Am. Soc. Mass Spectrom. 1994, 5, 58-63.

5. Chiarelli, M. P.; Wu, H.-P.; Antunes, A. M.; Branco, P. S. Product Ion Studies of Some Novel Arylamine Adducts of Deoxyguanosine by Matrix-Assisted Laser Desorption/Ionization and Post-Source Decay. Rapid Commun. Mass Spectrom. 1999, 13, 2004-2010.

6. Lay, J. O., Jr.; Gross, M. L.; Zwinselman, J. J.; Nibbering, N. M. M. A Field Ionization and Collisionally Activated Dissociation/Charge Stripping Study of Some $\left[\mathrm{C}_{9} \mathrm{H}_{10}\right]^{+}$ions. Org. Mass Spectrom. 1983, 18, 16-21.

7. Jacobson, M. D.; Shapiro, R.; Underwood, G. R.; Broyde, S.; Verna, L.; Hingerty, B. E. Synthesis and Conformation of a Dinucleoside Monophosphate Modified by Aniline. Chem. Res. Toxicol. 1988, 1, 152-159.

8. Bryant, M. S.; Lay, J. O., Jr.; Chiarelli, M. P. Development of Fast Atom Bombardment Mass Spectral Methods for the
Identification of Carcinogen-Nucleoside Adducts. J. Am. Soc. Mass Spectrom. 1992, 3, 360-371.

9. Marques, M. M.; Mourato, L. L.; Amorim, M. T.; Santos, M. A.; Melchior, W. B., Jr.; Beland, F. A. Effect of Substitution Site Upon the Oxidation Potentials of Alkylanilines, the Mutagenicities of $\mathrm{N}$-Hydroxyalkylanilines, and the Conformations of Alkylaniline-DNA Adducts. Chem. Res. Toxicol. 1997, 10, 12661274.

10. Marques, M. M.; Mourato, L. L.; Santos, M. A.; Beland, F. A. Synthesis, Characterization, and Conformational Analysis of DNA Adducts from Methylated Anilines Present in Tobacco Smoke. Chem. Res. Toxicol. 1996, 9, 99-108108.

11. Branco, P. S.; Antunes, A. M.; Marques, M. M.; Chiarelli, M. P.; Lobo, A. M.; Prabhakar, S. New Syntheses of DNA Adducts from Methylated Anilines Present in Tobacco Smoke. Chem. Res. Toxicol. 1999, 12, 1223-1233.

12. Gonçalves, L. L.; Beland, F. A.; Marques, M. M. Synthesis, Characterization, and Comparative ${ }^{32}$ P-Postlabeling Efficiencies of 2,6-Dimethylaniline-DNA Adducts. Chem. Res. Toxicol. 2001, 14, 165-174.

13. Chiarelli, M. P.; Lay, J. O., Jr. Mass Spectrometry for the Analysis of Carcinogen-DNA Adducts. Mass Spectrom. Rev. 1993, 11, 447-493.

14. Wan, K. X.; Vidavsky, I.; Gross, M. L. Comparing Similar Spectra: From Similarity Index to Spectral Contrast Angle. J. Am. Soc. Mass Spectrom. 2002, 13, 85-88.

15. Drahos, L.; Vekey, K. Quantification of Isomeric Differences in Mass Spectra. Rapid Commun. Mass Spectrom. 1996, 10, 1309_ 1315.

16. Stein, S. E.; Scott, D. R. Optimization and Testing of Mass Spectra Library Searching Algorithms for Compound Identification. J. Am. Soc. Mass Spectrom. 1994, 5, 859-866.

17. Maga, J. A.; Johnson, D. L.; Morini, G. Major Volatiles in Toasted Sesame Oil. J. Food Lipids 1995, 4, 259-268.

18. Gatlin, C. L.; Eng, J. K.; Cross, S. T.; Detter, J. C.; Yates, J.R. III. Automated Identification of Amino Acid Sequence Variations in Proteins by HPLC/Microspray Tandem Mass Spectrometry. Anal. Chem. 2000, 72, 757-763. 